\title{
Exfoliative Cheilitis
}

Altaf Hussain Chalkoo', Nusrat Nazir Makroo², Gowhar Yaqub Peerzada ${ }^{3}$

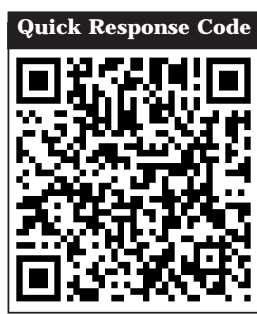

\section{doi: $10.5866 / 2016.8 .10056$}

${ }^{1}$ Professor and Head

${ }_{2 \& 3}$ Post Graduate Student

Department of Oral Medicine and Radiology,

Government Dental College,

Srinagar, J and K, India

\section{Article Info:}

Received: J anuary 10, 2016

Review Completed: February 11, 2016

Accepted: March 13, 2016

Available Online: May, 2016 (www.nacd.in)

(C) NAD, 2016 - All rights reserved

\section{Email for correspondence:}

draltafhussain321@gmail.com

\section{Introduction:}

Lip lesions can be manifestations of systemic diseases, a localized expression of dermatologic diseases or a localized condition of thelips. ${ }^{1}$ Cheilitis is classified into various types: angular cheilitis, actinic cheilitis, contact cheilitis, plasma cell cheilitis, cheilitis gland-ularis, cheilitis granulomatosa, exfoliative cheilitis and factitious cheilitis. ${ }^{2}$ Thorough clinical history, basiclaboratory tests and histopathologic evaluation are required to exclude other diseases. Exfoliative cheilitis is a rare disease which affects the vermilion border of thelips. It is characterized by unremitting production and desquamation of thick scales of keratin. ${ }^{1,3}$ Symptoms may include sensitivity and burning of the lips, and affected patients may avoid socializing due to the unsightly appearance of the lips. ${ }^{4}$ Although the cause is unknown, EC has been associated with underlying stress and anxiety as well as

\begin{abstract}
:
Exfoliative cheilitis (EC) defines a heterogeneous spectrum of chronic inflammatory processes characterized by a persisting crusting and desquamation of thelips. In majority of cases, cause of the disease is repetitive lip picking or biting which leads to excessive production and exfoliation of keratin and hence it is also termed as facticious cheilitis, however the disease it still considered as idiopathetic in origin. Factors such as stress and some psychiatric conditions are associated with the onset of the disease. This condition is disabling becauseesthetics and normal functions such as eating, speaking and smiling are compromised. The lack of specific treatment makes exfoliative cheilitis a chronic disease that radically affects a person's life. This report attempts to eliminate the possible differential diagnosis by proper investigations and reaching to correct diagnosis.
\end{abstract}

Key words: Exfoliative chilitis, anti-keratotic, Euciren ointment. 
HBsAg, anti-Hcv, Hiv, EBV, CMV serology were done which were negative. Patient was also subjected to pathergy test, montoux test, test for brucellosis and sarcodosis which were all negative. Patient also visited rheumatology department where she was investigated for autoimmune disease. Rh factor, C-reactive protein, Anti Nuclear antibody, anti ds DNA levels were within normal limits. Finally patient was referred to the department of Oral Medicine and Radiology to rule out any oral foci of infection. After performing all base line investigations and under all aseptic precautions an incisional biopsy was performed (Figure 2) and specimen was sent for histopathological examination and fungal culture.

Histopathological examination showed marked hyperkeratosis and parakeratosis, mild inflammatory infiltrate, without any significant characteristic feature and (Figure 3).

Fungal culture was also negative. From clinical examination, history and biopsy report it was clear that all features were suggestive of exfoliative cheilitis. When the patient was asked about the habit of lip picking or licking, sheadmitted that she had the habit but she had left the habit because of this condition now. Patient was emotionally disturbed because of the unesthetic appearance so a psychiatric consultation was done and patient was put on clonazipam $0.5 \mathrm{mg}$ at bed time meanwhile treatment of exfoliated cheilitis was commenced with local application of antikeratotic ointment $0.005 \%$ retinoic acid qid, Betamethasone $0.01 \%$ tid , euciren emollient cream with $10 \%$ urea and patient was advised to keep her lips always moist by using some lip baam in between and at the same time avoidance of lip licking was advised. A great improvement was seen after 15 days of treatment, upper lip was completely normal and great improvement was seen in lower lip. Crustations were resolved in middle portion, only small scales were present on lateral portion of lower lip (Figure 4 and 5). Same treatment regimen was continued and patient is on regular follow up however the condition has not completely resolved there are still periods of exacerbation.

\section{Discussion:}

Less than 200 cases of EC have been reported in the lit-erature (51 cases in the English literature) and their di-agnosis was totally based on history and clinical feature. ${ }^{7,8}$ Exfol iative cheilitis reportedly occurs more commonly in females., 9 The majority $(62 \%)$ of patients affected were younger than 30 years of age, many of whom were younger than 20 years of age same is the case with this patient. ${ }^{7}$ Clinician's knowledge of the clinical course of this disease is important for accurate diagnosis. Exfoliative cheilitis is an infrequently mentioned condition; details given about the course of this disease are inadequate. One of the leading hypotheses is that EC develops secondary to parafunctional activities, including lip licking and lip biting. Patients with psychologic conditions such as anxiety, depression, or obsessive compulsive disorder may have more persistent chronic parafunctional activities that can contribute to the development of EC. ${ }^{10}$ The present patient too was mentally disturbed however she had now left the habit of lip licking because of the pain and discomfort associated with even slight movement of lips and has lost weight because of loss of appetite and discomfort associated with normal functional activity of mouth. However, most patients treated with antidepressants showed improvement in, but no complete remission of the disease. In this case, patient's condition improved when she was on antidepressants, but she too had no complete relief. Raede and others discussed the possibility of cheilocandidosis. ${ }^{5}$ It involves compromised immunity or the presence of other obvious predisposing factors that cause candidal infection of the lips. The authors achieved successful resolution of such lesions with antifungal therapy. However, for people who have no specific predisposing factors, such as our patient and others Candida could not be isolated from the lesion nor did the condi-tion respond to antifungal therapy. ${ }^{11}$ Fungal colonies have been reported on histologic examination, but these appear to be superficial and are likely secondary findings rather than being pathogenic, as is typical with intraoral factitial/ frictional keratosis. ${ }^{12}$ Oral sepsis has also been implicated as a cause of ex-foliative cheilitis as in this case too patient had a very poor oral hygiene because it was dif-ficult to maintain good oral hygiene. ${ }^{13} \mathrm{Her}$ condition may be the result of multifactorial causes such as factitious habit, oral sepsis, associated with and aggravated by stress that resulted in clinical depression. Atopy, actinic damage, cheilitis granulomatosa, plasma cell cheilitis, contact dermatitis, discoid lupus erythematosus and neoplasia should be considered in the differential diagnosis of crusted and ulcerated lesions of the lip. ${ }^{14}$ Thorough clinical history, basic laboratory tests and histopathologic evaluation are required to exclude other diseases. 


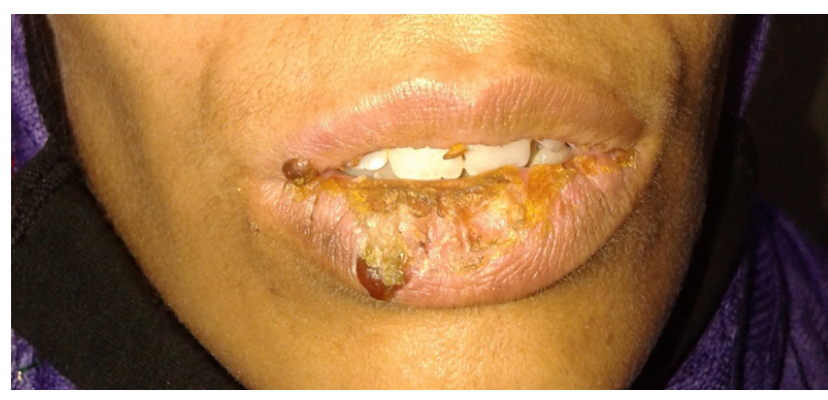

Figure 1: Clinical picture showing pretreatment condition of lip with marked crustation.

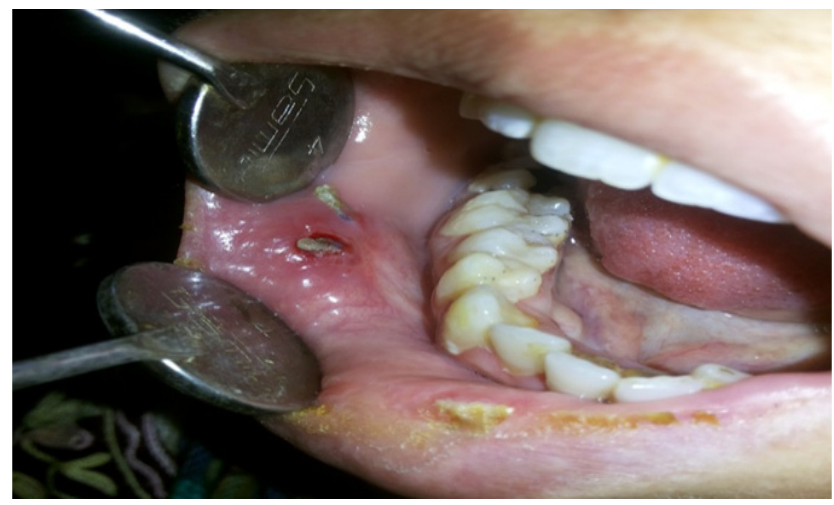

Figure 2: Site of incisional biopsy.

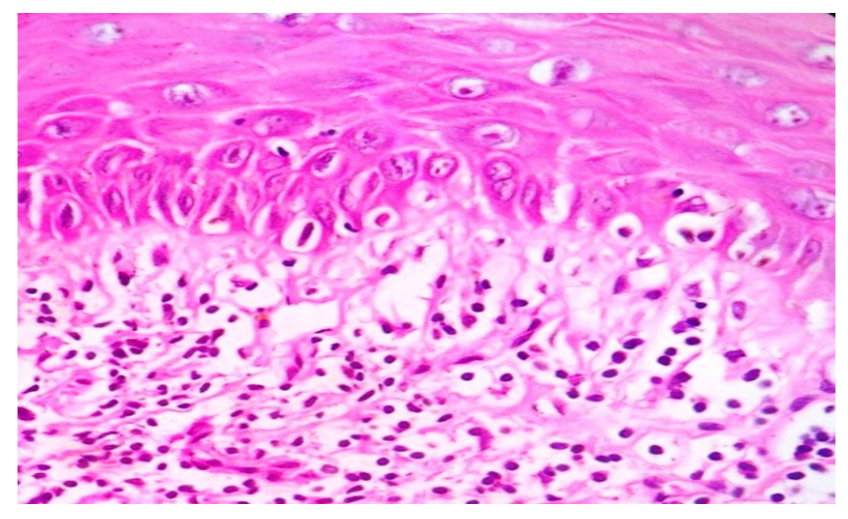

Figure 3: $H$ \& $E$ stained section showing non specific histopathological picture.

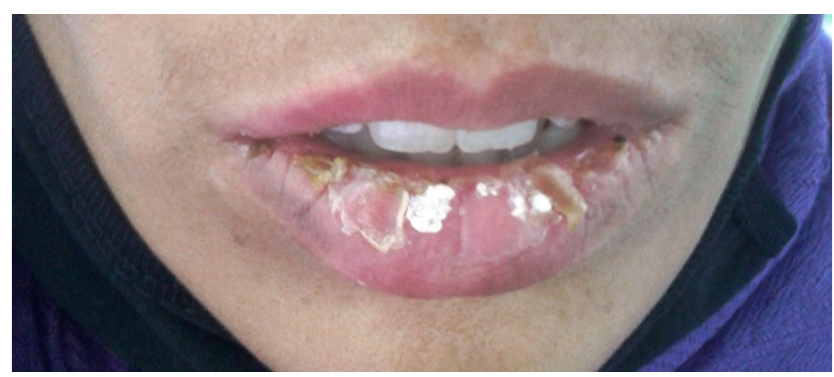

Figure 4: Clinical picture showing post treatment condition of lips after 15 days.

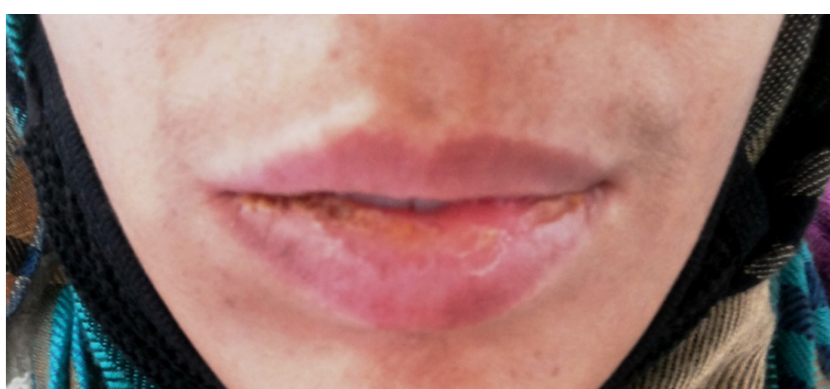

Figure 5: Clinical picture showing post treatment picture after 30 days.

Histologically, exfoliative cheilitis is characterized by marked hyperkeratosis and parakeratosis without significant inflammation. This could be reactive hyperplasia of the epidermis due to repetitive trauma in the form of regular picking of the lips. Trauma may induce epidermal proliferation resulting in parakeratosis and hyperkeratosis. ${ }^{14}$ So biopsy is the gold standard for diagnosis of this condition along with history of clinical course of the disease. Exfoliative cheilitis may resolve spontaneously but if persistent, it is usually refractory to treatment and difficult to manage. ${ }^{3,11}$ Previous studies for the most part have reported no benefit with antimicrobial agents (systemic and topical), corticosteroids (systemic and topical), petroleum jelly, sunscreen, and dietary supplements such as folic acid and iron. $3,4.8,9,15$ Systemic therapy with antidepressants including sertraline, fluvoxamine, diazepam, and amitriptyline led to at least partial improvement in all patients treated. ${ }^{3,7,15,16}$ Because none of these were controlled studies, it is unclear to what extent the efficacy associated with antidepressant medications was due to modification of parafunctional habits or simply to the natural course of the condition. EC can be easily confused with other dermatologic conditions that affect thelip, such as allergic contact cheilitis and atopic cheilitis. These lip conditions present with erythema, dryness, scaling, and fissuring. The most common causes are cosmetic materials, metals (commonly nickel), and dental materials. These can be distinguished from EC by a positive history of contact with the allergen/hapten, positive allergy or patch testing, and substantial response to elimination of the allergen. In the present case there was no history of use of new creams, toothpaste or cosmetic items around thelips before the problem began more over pathergy test also was negative. Avoidance of lip sucking or biting and use of lip moisturizers has been the mainstay of therapy. Psychiatric evaluation is essential for diagnosing any emotional stress that can exacerbate 
lesions ${ }^{19}$. In this report, pre-scription of clonazepam $0.5 \mathrm{mg}$ daily with antikeratotic cream Retinoic acid $0.005 \%$, Betamethasone $0.01 \%$, lip balm and Eucerin emollient cream (10\% urea) together caused a significant improvement. Urea is produced naturally in the skin and causes moisture ab-sorption and helps to rehydration of dry and scaly skin. Furthermore, urea in the Eucerin emollient cream ( $10 \%$ urea) penetrates to the horny layer of skin and increases the skin's capacity to absorb moisture. Eucerin covers skin surface as oil layer, which prevents water evapora-tion from this surface. Side effects of urea are skin irrita-tion such as burning, itching or erythema; however, in the present case there were no side effects. However these agents, combined with intensive counseling and psychotherapy have not yielded desired or satisfactory results. Therefore, besides the above mentioned agents various therapeutic modalities like cryosurgery, intralessional methotrexate injections have been tried in the past with variable outcomes. All of them were aimed at reducing excessive keratin and provides temporary and symptomatic relief. As the present patient had severe inconvenience and cosmetic disfigurement dueto the disease, we tried local antikeratotic agent, local steroid and eucerin ointment to reduce excessive epidermal proliferation. In this patient, significant improvement in scaling and erythema was seen. As such, it is difficult to be certain whether patient responded because of the anti-inflammatory effect of the ointment or because the ointment or moisturizing agents were emol lient and thus hel ped to keep the lips moist, protected the area from irritants, or increased the patients' awareness of the habit, hel ping them to reduce trauma to the site This case highlights the fact that exfoliative cheilitis is secondary to compulsive lip licking and psychiatric consultation along with above mention treatment regime can be used as therapeutic option in severely affected patients.

\section{Conclusion:}

Exfoliative cheilitis predominantly affects females under 30 years of age lower lip is affected more as compare to upper lip and typically follows a cyclic course characterized by normal or erythematous lips Given the scarcity of reports in the literature and the lack of a strong evidence base to guide therapy, management of EC will likely remain a challenge for clinicians and patients. Prospective multicenter trials are unlikely owing to the infrequent occurrence of EC. Larger case series from multiple centers would be helpful in understanding the natural course of EC and in identifying the most effective management strategies. No appropriate treatment has been identified for this condition because the cause remains unclear.

\section{References}

1. Rogers R S, 3rd, Bekic M. Diseases of the lips. SeminCutan Med Surg. 1997; 16:328-36.

2. Lynch MA, Brightman VJ , Greenberg MS, editors. Burket's oral medicine: diagnosis and treatment. 9th ed. Philadel phia: Lippincott-Raven Publishers; 1994.

3. Daley T D, Gupta A K. Exfoliative cheilitis. J Oral Pathol Med 1995; 24:177-9.

4. Leyland L, Field EA. Case report: exfoliative cheilitis managed with antidepressant medication. Dent Update 2004; 31:524-6.

5. Reade PC, Sim R. Exfoliative cheilitisda factitious disorder? Int J Oral Maxillofac Surg 1986; 15:313-7.

6. Neville BW, Damm DD, Allen CM, Bouquot J E. Physical and chemical injuries. In: Oral and Maxillofacial Pathology. 3rd ed. St Louis, MO: Saunders; 2009: 304-5.

7. Taniguchi S, KonoT. Exfoliative cheilitis: a case report and review of the literature. Dermatology 1998; 196(2):253-5.

8. Connolly M, Kennedy C. Exfoliative cheilitis successfully treated with topical tacrolimus. Br J Dermatol 2004; 151(1):241-2.

9. Tyldesley WR. Oral medicine. Oxford: Oxford Medical Publications; 1981. pg. 65.

10. Crotty $\mathrm{CP}$, Dicken $\mathrm{CH}$. Factitious lip crusting. Arch Dermatol 1981; 117:338-40.

11. Postlethwaite KR, Hendrickse NM. A case of exfoliative cheilitis. Br Dent J 1988; 165(1):23.

12. Woo SB, Lin D. Morsicatio mucosae orisda chronic oral frictional keratosis, not a leukoplakia. J Oral Maxillofac Surg 2009; 67:140-6.

13. Brooke RI. Exfoliative cheilitis. Oral Surg Oral Med Oral Pathol 1978; 45(1):52.

14. Aydin E, Gokoglu O, Ozcurumez G, Aydin H. Factitious cheilitis: A case report. J Med Case Reports 2008; 2:29.

15. Mani SA, Shareef BT. Exfoliative cheilitis: report of a case. J Can Dent Assoc 2007; 73:629-32.

16. Roveroni-F avaretto LH, Lodi KB, Almeida J D. Topical Calendula officinalis $L$. successfully treated exfoliative cheilitis: a case report. Cases J 2009; 2:9077.

17. Schena D, Fantuzzi F, Girolomoni G. Contact allergy in chronic eczematous lip dermatitis. Eur J Dermatol 2008; 18:688-92.

18. Freeman S, Stephens R. Cheilitis: analysis of 75 cases referred to a contact dermatitis clinic. AmJ Contact Dermat 1999; 10:198-200.

19. J eanmougin M, Civatte J Bertail MA. Factitious crusting cheilitis. Ann Dermatol Venereol 1984; 111:1007-11.

20. Netdoctor. Eucerin dry skin intensive $10 \%$ urea treatment cream. London: Netdoctor; 2015. Available from: http:// www.netdoctor. co.uk/skin-and-hair/medicines/eucerinintensive-10-per-cent-urea-treatment-cream.html. 